\title{
We're all computational biologists now...Next stop, the global brain?
}

\author{
Richard A. Jorgensen* \\ Laboratorio Nacional de Genómica para la Biodiversidad, Centro de Investigación y Estudios Avanzados, Irapuato, Mexico \\ ${ }^{*}$ Correspondence: rajorgensen@langebio.cinvestav.mx
}

In the late Twentieth Century the aphorism "We're all biologists now" entered the mainstream consciousness of biologists of all types. The new science of genomics had arrived on the scene, brashly and boldly challenging the many subdisciplines of biology to breach the walls that had served to separate them for decades. The result was a gradual, but eventually widespread realization that genomics comprised a new "commons" - a "marketplace" where the diverse cultures of biology could meet, learn each others' languages, exchange ideas, and form new ventures to exploit the astounding number of synergies that were rapidly becoming apparent.

Of course, we all know that the molecular biology revolution was the foundation of the genomics revolution - don't we? Molecular biology indeed made genomics possible, but it was wholly inadequate on its own to fully exploit the potential for genomics to revolutionize biology. In fact, most molecular biologists found themselves woefully unprepared for the new revolution that was beginning to emerge. Why? Ironically, it was molecular biologists who had instigated a rather messy divorce from what they viewed as the tradition-bound sciences of ecology, evolution, taxonomy, and systematics. These brash, "young bucks" of molecular biology shook traditional biology to its foundation, triggering a tsunami of divisions of biology departments into two distinct types of entities: new, reductionist-driven departments centered on molecular biology and biochemistry and more traditional, holistic departments centered on ecology, evolution, and biodiversity. (A few notable exceptions, such as Indiana University, successfully maintained a broad-based, integrated Biology Department.)

As a graduate student in the mid-70s, I remember quite well a rather cocksure postdoc dismissively stating that "we already know all the names of the weeds..."
"Weeds," of course was a dismissive code word for "flora" or "biota" - and there was no need to expend valuable resources on "That"! Such ignorance and arrogance seems shocking today, of course, but at the time it was not at all uncommon. Another common maxim among molecular biologists at the time was: "If you need to use statistics, you should design a different experiment!" This was said with emphasis and an air of certainty. Biology was being reduced to finding qualitative "yes" or "no" answers, and thus choosing to ask only those questions that could be so answered. This approach was considered to be "elegant." I think I exaggerate only slightly in saying that this was biology's "McCarthy Era" 1 . Of course, having been trained as a molecular biologist, I have to accept my share of responsibility to the extent that I might have furthered or tolerated such narrow views at the time.

The discipline of genetics was unique in that it had one foot on each side of the divide, stretching but not breaking, and it served as a bridge between the two cultures for those willing to traverse it. Genetics, of course, became permeated by molecular biology, first creating the field of molecular genetics, which explored the molecular nature of the gene and its expression. Later, molecular approaches were the foundation for a revolution in population and quantitative genetics, leading eventually to association genetics, which explores phenotype-genotype relationships across the genome. And as whole genome sequencing laid open the details of entire genomes, it gave birth to a new genetics subdiscipline that quickly came to be known as genomics.

Together, genetics, molecular biology, and genomics greatly expanded the means and scope for exploration of new territo-

${ }^{1}$ Senator Joseph McCarthy of Wisconsin was the public face and driving force of the 1950's movement to root out, expose, and punish "un-American activities." ries across diverse fields of biology, from biochemistry and biophysics to evolution and ecology, and became a unifying force, thus leading to the phrase "we're all biologists now," meaning that we are no longer merely cell biologists, physiologists, ecologists, or evolutionary biologists; rather, we are now simply biologists. The many molecular biologists who now called themselves genomicists began to arrive on the shores of what many of them imagined to be relatively unknown New Worlds, only to discover that they were already inhabited by sophisticated, if foreign cultures. Though misunderstandings and conflicts arose, this time a complex dance of diverse interactions ultimately bred mutual respect and new unions (collaborations) of almost any imaginable type, a process that is really only just beginning.

The current game-changer is the extraordinarily massive generation of new data, not only genomic data, but all the many forms of data needed to solve major biological problems, including images (from the cellular level to the ecosystem level), environmental data (from the microscopic to the ecosystem level), and survey data on the organismic composition of communities and ecosystems. Yes, even taxonomy is at the center of modern biology, the names of all those "weeds" having become hugely important for understanding fundamental principles of biology at the ecosystem and regional levels. Each of these types of data presents a tremendous challenge, necessitating new ways of cataloging, analyzing, and making sense of (understanding) these diverse types of overlapping and interrelating datasets.

Thus, the main force now beginning to reorganize biology and reorient biologists is computation, drawing on the diverse disciplines of computer science, information science, physics, mathematics, and statistics and giving rise to the "new" field of computational biology. Of course, the computational 
sciences have long been an important part of biology, extinct species of "yes-no" molecular biologists notwithstanding.

Genetics, of course, has always been rooted in statistics, beginning with both Mendel and Galston. The great Evolutionary Synthesis of the mid-Twentieth century, bringing together genetics and evolutionary biology, depended heavily on the mathematical genius of Haldane, Fisher, and Wright. Molecularly trained biologists interested in investigating systems and networks have been pleased and fortunate to find that ecologists had already been applying engineering principles to biology for decades, as had organismal biologists to individual organisms. Genomicists interested in comparative research were excited to discover that for many years phylogeneticists had been developing sophisticated algorithms and models to describe the Tree of Life and its implications for understanding the extraordinarily diverse Origins of Species. Through it all, genetics has been, and will continue to be at the center, accepting, embracing, and ultimately subsuming the newcomers, from molecular biology to genomics to computational biology.

If we are all becoming computational biologists (and indeed we are, whether all of us want to admit it or not), then we must recognize and celebrate the fact that not only does Biology exploit the computational sciences, but Biology IS a computational science.

The implications of widespread acceptance and understanding of this simple statement of fact will be revolutionary. We all like to believe and say that the Twenty-first Century is the Biology Century, and indeed it is. However, it is the union of biology with the computational sciences that will make it so - with genetics, that quiescent center out of which all biology develops and evolves, again serving as a bridge.

The story does not merely end here, all warm and fuzzy. Many believe that the central challenge of our times is nothing less than the sustainability of our Biosphere and our place in it: can we learn how to meet our needs today without compromising the ability of future generations to meet their needs? There can be no question that acceptance and understanding of the fact that Biology IS now a computational science will be an essential part of meeting the challenge of learning what it is going to take to develop and manage a sustainable Biosphere.

How are we going to solve this great challenge? I think it is becoming obvious that a good part of the solution lies in the emergent properties of the MetaWeb (also known as the Relationship Web), which is beginning to take form through the integration of the "Semantic Web" - which connects Knowledge - with the "Social Software" a diverse set of tools that connects People. Connecting knowledge and people by means of the MetaWeb is inevitably leading to emergence of a higher order capabilities, collectively referred to as the "Global Brain" (Nova Spivak, www.novaspivak.com).

Computational biology is essential for, and at the center of the Global Brain metaphor. Without computational biology the
Global Brain can have no temporal lobe (necessary for categorizing objects and entities), no parietal lobe (necessary for integrating disparate types of information to allow understanding of concepts), and no frontal lobe (necessary for interacting with and reacting to our environment, making associations and judgments, and assigning meaning).

Global Brain seems an apt metaphor for what computational biology is beginning to create. The implication lying within this metaphor is that humanity is participating in the evolution of a new form and expression of our collective conscious, as well as our collective conscience, driven by the ongoing emergence of the MetaWeb.

We are all computational biologists now, and we are beginning to integrate into the Global Brain. Will we do so with forethought and insight? Will we use it only to further our own selfish interests and those of our "tribe," or will we choose to use it for the benefit of the whole of humanity as intelligent partners in a Sustainable Biosphere? Which path will you choose?

Received: 30 August 2011; accepted: 15 September 2011; published online: 11 October 2011.

Citation: Jorgensen RA (2011) We're all computational biologists now... Next stop, the global brain? Front. Gene. 2:68. doi: 10.3389/fgene. 2011.00068

This article was submitted to Frontiers in Plant Genetics and Genomics, a specialty of Frontiers in Genetics.

Copyright $\odot 2011$ Jorgensen. This is an open-access article subject to a non-exclusive license between the authors and Frontiers Media SA, which permits use, distribution and reproduction in other forums, provided the original authors and source are credited and other Frontiers conditions are complied with. 